\title{
$\alpha$-Lithioenamines by chlorine-lithium exchange: versatile acyl anion equivalents
}

\author{
Miguel Yus,* Javier Ortiz, and Carmen Nájera \\ Departamento de Química Orgánica, Facultad de Ciencias, Universidad de Alicante, \\ Apdo. 99, 03080 Alicante (Spain) \\ E-mail:yus@ua.es
}

Dedicated to Professor Marcial Moreno-Mañas on occasion of his 60 birthday

(received 25 Oct 01; accepted 30 Jan 02; published on the web 07 Feb 02)

\begin{abstract}
The reaction of $\alpha$-chloroenamines 1 with an excess of lithium and a catalytic amount of 4,4'-ditert-butylbiphenyl (DTBB) in THF at $-90{ }^{\circ} \mathrm{C}$ followed by reaction with an electrophile $\left(\mathrm{D}_{2} \mathrm{O}\right.$, $\mathrm{Me}_{3} \mathrm{SiCl}, \mathrm{PhCOCH}=\mathrm{CHPh} / \mathrm{BF}_{3}, \mathrm{CO}_{2}, \mathrm{CyNCO}$ ) and final hydrolysis gave the expected functionalized enamines 2. In the case of aldehydes ( $t$ - BuCHO, $n-\mathrm{C}_{5} \mathrm{H}_{11} \mathrm{CHO}, \mathrm{PhCHO}$ ) it was necessary to perform the lithiation in the presence of the electrophile (Barbier conditions) at $40{ }^{\circ} \mathrm{C}$. Hydrolysis of the obtained enamines either with silica gel or with hydrochloric acid yielded the expected functionalized ketones $\mathbf{3}$.
\end{abstract}

Keywords: Chlorine-lithium exchange, acyl anion equivalent, $\alpha$-lithioenamine, ketones, $\alpha$ chloroenamines

\section{Introduction}

Acyl metals are interesting intermediates in synthetic organic chemistry because they are able to transfer the acyl functionality to electrophilic reagents, ${ }^{1}$ this reaction being a typical example of reactivity umpolung. ${ }^{2}$ The corresponding lithium derivatives (I) are very reactive and unstable, and have been prepared at low temperature by (a) carbonylation of some alkyllithium reagents, ${ }^{3}$ (b) tellurium-lithium exchange, ${ }^{4}$ and (c) in especial cases by direct deprotonation of crowded aldehydes. ${ }^{5}$ Due to the difficulty to generate acyllithium reagents, other alternatives including mainly the use of lithiated 1,3-dithianes and related systems (II), ${ }^{6}$ lithiated imines (III), ${ }^{7,8}$ and other lithiated compounds ${ }^{9}$ have been developed. Among this last group, are lithiated enol ethers (IV), ${ }^{10}$ which can be prepared by direct deprotonation. However, the corresponding enamines cannot be lithiated directly at the $\alpha$-position (to give intermediates of type $\mathbf{V}$ ) due to their low acidity. For this reason, we thought that compounds of type $\mathbf{V}$ could be prepared from the corresponding $\alpha$-chloroenamines by a chlorine-lithium exchange using an arene-catalyzed 
lithiation, ${ }^{11-13}$ a methodology employed extensively in our group during the last decade for the generation of organolithium compounds under very mild reaction conditions. ${ }^{14}$ Thus, this technology has allowed us (a) to generate simple organolithiums from non-halogenated materials, ${ }^{15}$ (b) to prepare functionalized organolithium compounds ${ }^{16}$ by chlorine-lithium exchange ${ }^{17}$ or by ring opening of heterocycles, ${ }^{18}$ (c) to generate polylithium synthons, ${ }^{19}$ and (d) to activate other metals, ${ }^{20}$ especially nickel. ${ }^{21}$ In many cases, for very unstable intermediates, it is necessary to perform the lithiation in the presence of the electrophilic reagent (Barbier type conditions). ${ }^{22}$ In this paper we report on the application of the mentioned arene-catalyzed lithiation to prepare $\alpha$-lithioenamines of the type $\mathbf{V}$ by chlorine-lithium exchange. ${ }^{23}$<smiles>CC(=O)I</smiles><smiles>CC1([AlH2])SCCCS1</smiles><smiles>[R]N=C(C)[AlH2]</smiles>

III<smiles>[R]OC(=C)Cl</smiles>

IV<smiles>[R20]C(=C)Cl</smiles>

\section{Results and Discussion}

The reaction of $\alpha$-chloroenamines 1 (prepared by treatment of the corresponding acyl chlorides with dimethylamine or pyrrolidine followed by reaction of the formed amide with phosphorus oxychloride $)^{24}$ with an excess of lithium (1:7 molar ratio) and a catalytic amount of 4,4'-di-tertbutylbiphenyl (DTBB; 1:0.05 molar ratio) in THF at $-90^{\circ} \mathrm{C}$ led, after $50 \mathrm{~min}$, to a solution of the corresponding intermediate VI. Treatment of this species with $\mathrm{D}_{2} \mathrm{O}$ or $\mathrm{Me}_{3} \mathrm{SiCl}$ at temperatures ranging between -90 and $-40{ }^{\circ} \mathrm{C}$ gave, after hydrolysis with water, the expected compounds 2aa,ba or 2ab, respectively (Scheme 1, Method A, and Table 1, entries 1, 2 and 5). When carbonyl compounds were used as electrophiles, the best results were obtained performing the reaction in the presence of the electrophile (Method B). Thus, for pivalaldehyde, the same lithiation mixture described above was used with starting materials $\mathbf{1}$ in the presence of the aldehyde at $-40{ }^{\circ} \mathrm{C}$ for $1 \mathrm{~h}$ and, after hydrolysis, the expected compounds 2 ac and 2 bc were isolated (Scheme 1 and Table 1, entries 3 and 6). When benzaldehyde was used in the same reaction (Method B), aminoketone $\mathbf{2}^{\prime}$ ad was obtained, resulting from an isomerization of the expected enaminoalcohol (2ad) under the reaction conditions used (Scheme 1 and Table 1, entry 4).<smiles>[R]C([R2])=C([R])Cl</smiles>

1a: $\mathrm{R}_{2}^{1}=\left(\mathrm{CH}_{2}\right)_{4}, \mathrm{R}_{2}^{2}=\mathrm{Me}_{2}$

1b : $\mathrm{R}_{2}{ }_{2}=\mathrm{Me}_{2}, \mathrm{R}_{2}{ }_{2}=\left(\mathrm{CH}_{2}\right)_{4}$

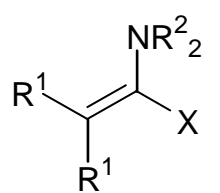

2

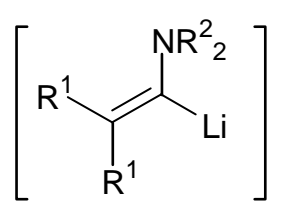

VI

Scheme 1. (a) Li, DTBB cat. (5 mol \%), THF, $-90{ }^{\circ} \mathrm{C}, 50 \mathrm{~min}$; (b) $\mathrm{E}^{+}=\mathrm{D}_{2} \mathrm{O}, \mathrm{Me}_{3} \mathrm{SiCl}$, $\mathrm{PhCOCH}=\mathrm{CHPh} / \mathrm{BF}_{3} \cdot \mathrm{OEt}_{2}, \mathrm{CO}_{2}, \mathrm{CyNCO},-90$ to $-40{ }^{\circ} \mathrm{C}$; (c) $\mathrm{H}_{2} \mathrm{O},-40$ to $20{ }^{\circ} \mathrm{C}$; (d) $\mathrm{Li}$, DTBB cat. $(5 \mathrm{~mol} \%), \mathrm{E}^{+}=t-\mathrm{BuCHO}, n-\mathrm{C}_{5} \mathrm{H}_{11} \mathrm{CHO}, \mathrm{PhCHO}$, THF, $-40{ }^{\circ} \mathrm{C}, 1 \mathrm{~h}$. 

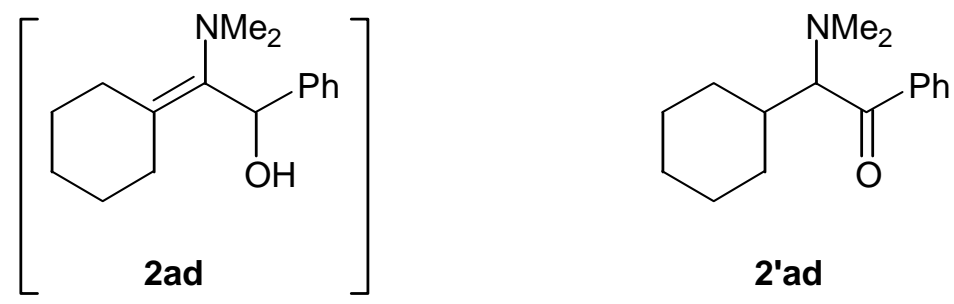

Table 1. Preparation of functionalized enamines 2

\begin{tabular}{ccccccccc}
\hline Entry & Starting & Electrophile & Method $^{a}$ & \multicolumn{5}{c}{ Product 2 $^{b}$} \\
\hline & material & $\mathrm{E}^{+}$ & & $\mathrm{No}$ & $\mathrm{R}_{2}{ }_{2}$ & $\mathrm{R}_{2}$ & $\mathrm{X}$ & Yield (\%) $^{c}$ \\
\hline 1 & $\mathbf{1 a}$ & $\mathrm{D}_{2} \mathrm{O}$ & $\mathrm{A}$ & $\mathbf{2 a a}$ & $\left(\mathrm{CH}_{2}\right)_{4}$ & $\mathrm{Me}_{2}$ & $\mathrm{D}$ & $81^{d}$ \\
2 & $\mathbf{1 a}$ & $\mathrm{Me}_{3} \mathrm{SiCl}$ & $\mathrm{A}$ & $\mathbf{2 a b}$ & $\left(\mathrm{CH}_{2}\right)_{4}$ & $\mathrm{Me}_{2}$ & $\mathrm{Me}_{3} \mathrm{Si}$ & 71 \\
3 & $\mathbf{1 a}$ & $t-\mathrm{BuCHO}$ & $\mathrm{B}$ & $\mathbf{2 a c}$ & $\left(\mathrm{CH}_{2}\right)_{4}$ & $\mathrm{Me}_{2}$ & $t-\mathrm{BuCHOH}$ & 62 \\
4 & $\mathbf{1 a}$ & $\mathrm{PhCHO}$ & $\mathrm{B}$ & $\mathbf{2} \mathbf{a d}$ & $\left(\mathrm{CH}_{2}\right)_{4}$ & $\mathrm{Me}_{2}$ & $\mathrm{PhCHOH}$ & 55 \\
5 & $\mathbf{1 b}$ & $\mathrm{D}_{2} \mathrm{O}$ & $\mathrm{A}$ & $\mathbf{2 b a}$ & $\mathrm{Me}_{2}$ & $\left(\mathrm{CH}_{2}\right)_{4}$ & $\mathrm{D}$ & $80^{e}$ \\
6 & $\mathbf{1 b}$ & $t-\mathrm{BuCHO}$ & $\mathrm{B}$ & $\mathbf{2 b c}$ & $\mathrm{Me}_{2}$ & $\left(\mathrm{CH}_{2}\right)_{4}$ & $t-\mathrm{BuCHOH}$ & 44 \\
\hline
\end{tabular}

${ }^{a}$ Method A: two-step reaction; Method B: Barbier-type conditions.

${ }^{b}$ All products 2 (or 2') were $>90 \%$ pure (GLC and/or $300 \mathrm{MHz}{ }^{1} \mathrm{H}$ NMR).

${ }^{c}$ Isolated crude yield based on the starting chloro enamine $\mathbf{1}$.

${ }^{d}>90 \%$ Deuterium incorporation from mass spectrometry. 
<smiles>CC(C)(C)C(O)C(C)(C)C</smiles>

3ac $(52 \%)^{a}$<smiles>CCCCCC(C)O</smiles>

3ae $(41 \%)^{a, b}$<smiles>CCCCCC(=O)C(O)C1CCCCC1</smiles>

3'ae<smiles>O=C(C1CCCCC1)C(O)(/C=C/c1ccccc1)C1CCCCC1</smiles>

3af

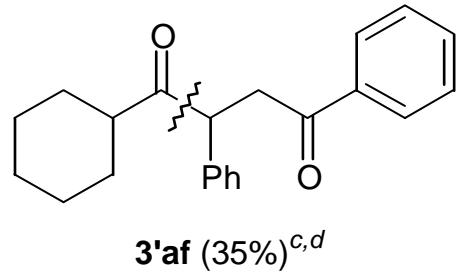

3'af $(35 \%)^{c, d}$<smiles>CC(C)C(=O)C(O)C(C)(C)C</smiles>

3 bc $(27 \%)^{a}$<smiles>COC(=O)C(=O)C1CCCCC1</smiles>

$3 \operatorname{ag}(32 \%)^{c, e}$<smiles>CC(C)C(=O)C(=O)NC1CCCCC1</smiles>

3bh $(36 \%)^{c}$

Chart 1. Preparation of compounds 3

Isolated yields after column chromatography (silica gel, hexane/ethyl acetate) based on the starting chloro enamine $\mathbf{1}$ (overall yield)

${ }^{a}$ Method B.

${ }^{b}$ Crude yield before chromatographic purification (see text).

${ }^{c}$ Method A.

${ }^{d} \mathrm{BF}_{3} \cdot \mathrm{OEt}_{2}$ was used to activate chalcone (1:1 molar ratio).

${ }^{e}$ Esterification of the corresponding hydroxy acid was performed with methanol under $\mathrm{H}_{2} \mathrm{SO}_{4}$ catalysis.

In the second part of this study we performed the hydrolysis of functionalized enamines 2 , either after their isolation (from compounds $\mathbf{2 a c}$ and $\mathbf{2 b c}$, with silica gel for $20 \mathrm{~h}$ ) or in an onepot procedure without isolation of the corresponding compound 2 (with $2 \mathrm{M}$ hydrochloric acid for $1 \mathrm{~h}$ ), to give compounds 3 (Chart 1). For the one-pot procedure, the sequential method (Method A) was used in all cases (with chalcone, carbon dioxide or cyclohexyl isocyanate) except for the case of hexanal, in which the Barbier technology (Method B) was employed. It is worthy to note that in the reaction with chalcone, activation of the electrophile with boron trifluoride was necessary, ${ }^{25}$ obtaining only the corresponding 1,2-addition product 3af, which under the reaction conditions isomerized to the diketone $\mathbf{3}^{\prime} \mathbf{a f}$ (Chart 1). On the other hand, when crude compound 3ae was submitted to purification by column chromatography (silica gel, hexane/ethyl acetate), an isomerization took place giving a 3:1 mixture of compounds 3ae:3'ae 
(300 MHz ${ }^{1} \mathrm{H}$ NMR; Chart 1). The reaction of chloroenamine 1a with carbon dioxide gave a ketoacid, which was isolated and purified after conversion into the corresponding methyl ester 3 ag by treatment with methanol under acid catalysis (Chart 1). Finally, the use of cyclohexyl isocyanate as electrophile afforded the expected ketoamides 3ah and $\mathbf{3 b h}$ (Chart 1).

As a conclusion, we have reported here the preparation of $\alpha$-lithioenamines by a chlorinelithium exchange, which represent a versatile class of acyllithium synthons, which by reaction with different electrophiles and final hydrolysis allowed the preparation of functionalized ketones resulting from the transfer of the acyl moiety to the electrophile.

\section{Experimental Section}

General Procedures. For general information, see references 17 and 26.

Preparation of starting chloroenamines 1. Chloroenamines 1 were prepared according to the procedure described in the literature ${ }^{24}$ and characterized spectroscopically as follows.

$\boldsymbol{N}$-[Chloro(cyclohexylidene)methyl]- $\boldsymbol{N}, \boldsymbol{N}$-dimethylamine (1a). ${ }^{24}$ Oil; IR $\left(\mathrm{CH}_{2} \mathrm{Cl}_{2}\right) \vee 1665 \mathrm{~cm}^{-1}$ $(\mathrm{C}=\mathrm{C}) ;{ }^{1} \mathrm{H}$ NMR $\left(\mathrm{CDCl}_{3}\right) \delta$ 1.40-1.60 [6H, m, $\left.\mathrm{CH}_{2}\left(\mathrm{CH}_{2}\right)_{3} \mathrm{CH}_{2}\right], 2.20-2.40\left[4 \mathrm{H}, \mathrm{m},\left(\mathrm{CH}_{2}\right)_{2} \mathrm{C}\right.$ ], $2.40\left[6 \mathrm{H}, \mathrm{s},\left(\mathrm{CH}_{3}\right)_{2} \mathrm{~N}\right] ;{ }^{13} \mathrm{C}-\mathrm{NMR}\left(\mathrm{CDCl}_{3}\right) \delta 26.6,27.3(2 \mathrm{C}), 30.6(2 \mathrm{C})\left(5 \times \mathrm{CH}_{2}\right), 42.9$ [N( $\left.\left(\mathrm{CH}_{3}\right)_{2}\right], 130.9\left(\mathrm{CCH}_{2}\right), 138.1(\mathrm{CCl})$; LRMS m/z $173\left(\mathrm{M}^{+}, 60 \%\right), 158(23), 138(100)$.

1-(1-Chloro-2-methyl-1-propenyl)pyrrolidine (1b). ${ }^{24} \mathrm{Oil} ; \mathrm{IR}\left(\mathrm{CH}_{2} \mathrm{Cl}_{2}\right) \vee 1645 \mathrm{~cm}^{-1}(\mathrm{C}=\mathrm{C}) ;{ }^{1} \mathrm{H}$ NMR $\left(\mathrm{CDCl}_{3}\right) \delta 1.78\left[6 \mathrm{H}, \mathrm{s},\left(\mathrm{CH}_{3}\right)_{2} \mathrm{~N}\right], 1.78-1.84\left(4 \mathrm{H}, \mathrm{m}, \mathrm{NCH}_{2} \mathrm{CH}_{2}\right), 2.80-2.90[4 \mathrm{H}, \mathrm{m}$, $\left.\mathrm{N}\left(\mathrm{CH}_{2}\right)_{2}\right] ;{ }^{13} \mathrm{C}-\mathrm{NMR}\left(\mathrm{CDCl}_{3}\right) \delta 21.1\left(\mathrm{CH}_{3}\right), 25.8\left[\mathrm{~N}\left(\mathrm{CH}_{2} \mathrm{CH}_{2}\right)_{2}\right], 51.3\left[\mathrm{~N}\left(\mathrm{CH}_{2} \mathrm{CH}_{2}\right)_{2}\right], 126.1$ [C( $\left.\left(\mathrm{CH}_{3}\right)_{2}\right], 138.2(\mathrm{CCl})$; LRMS m/z $159\left(\mathrm{M}^{+}, 45\right), 144$ (9), 131 (9), 130 (4), 124 (100).

DTBB-Catalysed lithiation of $\alpha$-chloroenamines 1 and reaction with electrophiles (Method A). Isolation of compounds $2 \mathrm{aa}, 2 \mathrm{ab}$, and $2 \mathrm{ba}$. General procedure

To a green suspension of lithium powder $(100 \mathrm{mg}, 14 \mathrm{mmol})$ and DTBB $(26 \mathrm{mg}, 0.10 \mathrm{mmol})$ in THF $(6 \mathrm{~mL})$ at $-90^{\circ} \mathrm{C}$ under an argon atmosphere, was slowly added (ca. $50 \mathrm{~min}$ ) a solution of the starting material $\mathbf{1 a}$ o $\mathbf{1 b}(2 \mathrm{mmol})$ in THF $(4 \mathrm{~mL})$. Once the green colour was recovered the corresponding electrophile was added. In the case of deuterium oxide, an excess $(500 \mu \mathrm{L})$ was dropped over the mixture and then hydrolysed with water at $-90{ }^{\circ} \mathrm{C}$. When chlorotrimethylsilane was used, its solution ( $2 \mathrm{mmol})$ in THF ( $2 \mathrm{~mL}$ ) was added, allowing the temperature to rise to $40^{\circ} \mathrm{C}$. Then the reaction mixture was hydrolyzed with water. The crude mixture was extracted with ether $(3 \times 10 \mathrm{ml})$, the organic layer washed with water, dried over anhydrous $\mathrm{Na}_{2} \mathrm{SO}_{4}$ and the solvents evaporated (15 Torr) to give the crude title compounds, which could not be purified by column chromatography on silica gel. Yields are included in Table 1. Spectroscopic and analytical data follow.

Cyclohexylidenedeutero- $\boldsymbol{N}, \boldsymbol{N}$-dimethylmetanamine (2aa). Oil; ${ }^{1} \mathrm{H}-\mathrm{NMR}\left(\mathrm{CDCl}_{3}\right) \delta 1.40-1.60$, 1.60-1.70 (4H, 2H, respectively, 2m, $\left.\mathrm{CH}_{2}\left(\mathrm{CH}_{2}\right)_{3} \mathrm{CH}_{2}\right), 1.90-2.00,2.15-2.25(2 \mathrm{H}, 2 \mathrm{H}, 2 \mathrm{~m}$, $\left.\mathrm{CH}_{2} \mathrm{CCH}_{2}\right), 2.48\left[6 \mathrm{H}, \mathrm{s},\left(\mathrm{CH}_{3}\right)_{2} \mathrm{~N}\right] ;{ }^{13} \mathrm{C}-\mathrm{NMR}\left(\mathrm{CDCl}_{3}\right) \delta 26.7,27.15,27.7,28.35,33.35(5 \times$ 
$\left.\mathrm{CH}_{2}\right), 45.7\left[\left(\mathrm{CH}_{3}\right)_{2} \mathrm{~N}\right], 128.05\left[\mathrm{C}\left(\mathrm{CH}_{2}\right)_{2}\right], 133.75(1 \mathrm{C}, \mathrm{t}, \mathrm{J}=25.0, \mathrm{CD}) ; \mathrm{LRMS} \mathrm{m} / \mathrm{z} 140\left(\mathrm{M}^{+}\right.$, 71\%), 139 (32), 125 (37), 111 (70), 99 (14), 97 (46), 85 (55), 83 (53), 72 (47), 59 (62), 43 (100).

Cyclohexylidene- $N, N$-dimethyl(trimethylsilyl)metanamine (2ab). Oil; ${ }^{1} \mathrm{H}-\mathrm{NMR}\left(\mathrm{CDCl}_{3}\right) \delta$ 0.14 [9H, s, $\left(\mathrm{CH}_{3}\right)_{3} \mathrm{Si}$ ] , 1.40-1.55 (6H, m, $\left.\mathrm{CH}_{2}\left(\mathrm{CH}_{2}\right)_{3} \mathrm{CH}_{2}\right), 2.05-2.15,2.20-2.30$ (2H, 2H, 2m, $\left.\mathrm{CH}_{2} \mathrm{CCH}_{2}\right), 2.50\left[6 \mathrm{H}, \mathrm{s},\left(\mathrm{CH}_{3}\right)_{2} \mathrm{~N}\right] ;{ }^{13} \mathrm{C}-\mathrm{NMR}\left(\mathrm{CDCl}_{3}\right) \delta 2.3\left[3 \mathrm{C},\left(\mathrm{CH}_{3}\right)_{3} \mathrm{Si}\right], 26.95,28.328 .65$, 30.25, $32.9\left(5 \times \mathrm{CH}_{2}\right), 44.15\left[\left(\mathrm{CH}_{3}\right)_{2} \mathrm{~N}\right], 143.85(\mathrm{C}=\mathrm{CN}), 151.8(\mathrm{CN}) ; \mathrm{LRMS} \mathrm{m} / \mathrm{z} 211\left(\mathrm{M}^{+}, 37 \%\right)$, 196 (96), 138 (100), 114 (16), 73 (82), 67 (16), 59 (40), 42 (37).

1-(1-Deutero-2-methyl-1-propenyl)pyrrolidine (2ba). Oil; ${ }^{1} \mathrm{H}-\mathrm{NMR}\left(\mathrm{CDCl}_{3}\right) \delta 1.63,1.69(3 \mathrm{H}$, $3 \mathrm{H}$, respectively, $\left.2 \mathrm{~s}, 2 \times \mathrm{CH}_{3}\right), 1.75-1.85$ [4H, m, $\mathrm{CH}_{2}\left(\mathrm{CH}_{2}\right)_{2} \mathrm{CH}_{2} \mathrm{~N}$ ], 2.85-3.00 (4H, m, $\left.\mathrm{CH}_{2} \mathrm{NCH}_{2}\right) ;{ }^{13} \mathrm{C}-\mathrm{NMR}\left(\mathrm{CDCl}_{3}\right) \delta 17.85,22.9\left(2 \times \mathrm{CH}_{3}\right), 24.85\left[2 \mathrm{C}, \mathrm{CH}_{2}\left(\mathrm{CH}_{2}\right)_{2} \mathrm{CH}_{2} \mathrm{~N}\right], 53.6(2 \mathrm{C}$, $\left.\mathrm{CH}_{2} \mathrm{NCH}_{2}\right), 113.5\left[\mathrm{C}\left(\mathrm{CH}_{3}\right)_{2}\right], 134.15(1 \mathrm{C}, \mathrm{t}, J=25.6, \mathrm{CD})$; LRMS m/z $126\left(\mathrm{M}^{+}, 82 \%\right), 125$ (100), 111 (88), 97 (55), 85 (22), 83 (39), 70 (25), 69 (18), 56 (43), 55 (32), 43 (57), 42 (62).

DTBB-Catalyzed lithiation of $\alpha$-chloroenamines 1 under Barbier conditions (Method B). Isolation of compounds $2 \mathrm{ac}, 2$ 'ad and $2 \mathrm{bc}$. General procedure

To a green suspension of lithium powder (100 mg, $14 \mathrm{mmol})$ and DTBB (26 mg, $0.10 \mathrm{mmol})$ in THF (6 mL) was slowly added (ca. $40 \mathrm{~min})$ a solution of starting material 1 (2 mmol) and the corresponding electrophile (pivaladehyde, benzaldehyde or hexanal) in THF (4 mL) at $-40^{\circ} \mathrm{C}$ under an argon atmosphere. The reaction mixture was stirred for $20 \mathrm{~min}$ at the same temperature and then hydrolyzed with water $(10 \mathrm{~mL})$. After warming to room temperature the crude mixture was extracted with ether $(3 \times 20 \mathrm{ml})$, the organic layer was washed with water and dried with anhydrous $\mathrm{Na}_{2} \mathrm{SO}_{4}$. The solvents were evaporated (15 Torr) giving a residue, which was characterized in crude form in the case of the products $\mathbf{2} \mathbf{a c}$ and $\mathbf{2 b c}$ or, was purified by column chromatography (silica gel, hexane/ethyl acetate) affording pure title compound 2'ad. Yields are included in Table 1. Spectroscopic and analytical data follow.

1-Cyclohexylidene-3,3-dimethyl-1-(dimethylamino)-2-butanol (2ac). ${ }^{1} \mathrm{H}-\mathrm{NMR}\left(\mathrm{CDCl}_{3}\right) \delta$ $0.91\left[9 \mathrm{H}, \mathrm{s},\left(\mathrm{CH}_{3}\right)_{3} \mathrm{C}\right], 1.45-1.63\left[6 \mathrm{H}, \mathrm{m}, \mathrm{CH}_{2}\left(\mathrm{CH}_{2}\right)_{3} \mathrm{CH}_{2}\right], 2.00-2.25\left(4 \mathrm{H}, \mathrm{m}, \mathrm{CH}_{2} \mathrm{CCH}_{2}\right), 2.68$ $\left[6 \mathrm{H}, \mathrm{s},\left(\mathrm{CH}_{3}\right)_{2} \mathrm{~N}\right], 3.44(1 \mathrm{H}$, br s, OH$), 4.09(1 \mathrm{H}, \mathrm{s}, \mathrm{CHOH}) ;{ }^{13} \mathrm{C}-\mathrm{NMR}\left(\mathrm{CDCl}_{3}\right) \delta 26.75$ $\left[\left(\mathrm{CH}_{3}\right)_{3} \mathrm{C}\right], 26.55,26.75,27.4,27.65,31.5,\left(5 \times \mathrm{CH}_{2}\right), 30.65\left[\left(\mathrm{CH}_{3}\right)_{3} \mathrm{C}\right], 45.5$ [br s, $\left.\left(\mathrm{CH}_{3}\right)_{2} \mathrm{~N}\right]$, $76.15(\mathrm{CHOH}), 138.2,140.6(\mathrm{C}=\mathrm{C})$; LRMS m/z $225\left(\mathrm{M}^{+}, 15 \%\right), 210$ (10), 169 (46), 168 (88), 138 (100), 123 (11), 86 (23), 85 (12), 67 (23), 46 (46), 44 (54); HRMS m/z M M $^{+} 225.2133$, $\mathrm{C}_{14} \mathrm{H}_{27} \mathrm{NO}$ requires 225.2093.

Cyclohexyl-( $\boldsymbol{N}, \boldsymbol{N}$-dimethylamino)methyl phenyl ketone (2'ad). Oil; $R_{\mathrm{f}}=0.42$ (hexane/ethyl acetate:7/3); IR $v$ (film) $1672 \mathrm{~cm}^{-1}(\mathrm{C}=\mathrm{O}) ;{ }^{1} \mathrm{H}-\mathrm{NMR}\left(\mathrm{CDCl}_{3}\right) \delta 1.20-2.00\left(11 \mathrm{H}, \mathrm{m}, 5 \times \mathrm{CH}_{2}\right.$, $\left.\mathrm{CHCH}_{2}\right), 2.32\left[6 \mathrm{H}, \mathrm{s},\left(\mathrm{CH}_{3}\right)_{2} \mathrm{~N}\right], 3.96(1 \mathrm{H}, \mathrm{d}, J=9.8, \mathrm{CHN}), 7.42-7.59(3 \mathrm{H}, \mathrm{m}, \mathrm{ArH}), 7.95(2 \mathrm{H}$, $\mathrm{dd}, J=8.5,1.2, \mathrm{ArH}) ;{ }^{13} \mathrm{C}-\mathrm{NMR}\left(\mathrm{CDCl}_{3}\right) \delta 25.9,26.0,26.65,30.3,30.45\left(5 \times \mathrm{CH}_{2}\right), 37.05$ $\left(\mathrm{CHCH}_{2}\right), 41.6\left[\left(\mathrm{CH}_{3}\right)_{2} \mathrm{~N}\right], 70.1(\mathrm{CHN}), 127.85,128.6,132.8,139.6(4 \times \mathrm{ArC}), 201.55(\mathrm{C}=\mathrm{O})$; LRMS m/z 162 (M+ - cyclohexyl, 1.5\%), 141 (12), 140 (100), 95 (15), 77 (13), 58 (38), 36 (21), 44 (13); HRMS m/z M ${ }^{+}, 245.1822, \mathrm{C}_{16} \mathrm{H}_{23} \mathrm{NO}$ requires 245.1780.

4-Tetrahydro-1H-1-pyrrolyl-2,2,5-trimethyl-4-hexen-3-ol (2bc). Oil; ${ }^{1} \mathrm{H}-\mathrm{NMR}\left(\mathrm{CDCl}_{3}\right) \delta$ $0.91\left[9 \mathrm{H}, \mathrm{s},\left(\mathrm{CH}_{3}\right)_{3} \mathrm{C}\right], 1.65,1.66\left[3 \mathrm{H}, 3 \mathrm{H}, 2 \mathrm{~s},\left(\mathrm{CH}_{3}\right)_{2} \mathrm{C}=\mathrm{C}\right], 1.80-1.95$ [4H, m, $\left.\mathrm{CH}_{2}\left(\mathrm{CH}_{2}\right)_{2} \mathrm{CH}_{2} \mathrm{~N}\right]$, 
2.95-3.10 (4H, m, $\left.\mathrm{CH}_{2} \mathrm{NCH}_{2}\right), 4.09(1 \mathrm{H}, \quad \mathrm{s}, \mathrm{CHOH}) ;{ }^{13} \mathrm{C}-\mathrm{NMR}\left(\mathrm{CDCl}_{3}\right) \quad \delta \quad 20.5,21.9$ $\left[\left(\mathrm{CH}_{3}\right)_{2} \mathrm{C}=\mathrm{C}\right], 26.05\left[2 \mathrm{C}, \mathrm{CH}_{2}\left(\mathrm{CH}_{2}\right)_{2} \mathrm{CH}_{2} \mathrm{~N}\right], 26.75\left[\left(\mathrm{CH}_{3}\right)_{3} \mathrm{C}\right], 37.25\left[\left(\mathrm{CH}_{3}\right)_{3} \mathrm{C}\right], 53.05(2 \mathrm{C}$, $\left.\mathrm{CH}_{2} \mathrm{NCH}_{2}\right), 76.9(\mathrm{CHOH}), 129.45\left[\mathrm{C}=\mathrm{C}\left(\mathrm{CH}_{3}\right)_{2}\right], 140.75(\mathrm{CN})$; LRMS m/z $211\left(\mathrm{M}^{+}, 10 \%\right), 156$ (24), 155 (72), 139 (18), 126 (42), 124 (88), 112 (11), 84 (15), 70 (86), 57 (21), 55 (48), 43 (40); HRMS $m / z \mathrm{M}^{+}, 211.1939, \mathrm{C}_{13} \mathrm{H}_{25} \mathrm{NO}$ requires 211.1936.

Silica gel-promoted hydrolysis of in situ generated (Method B) compounds 2ac, 2ae and 2bc. Isolation of compounds $3 \mathrm{ac}, 3 \mathrm{ae} / 3$ 'ae and $3 \mathrm{bc}$. General procedure

Once title compounds 2 were prepared as it was above describe (Method B), the crude product was adsorbed on silica gel $(5 \mathrm{~g})$ for $20 \mathrm{~h}$. The resulting mixture was then extracted with ether, the solvent evaporated and the residue purified by column chromatography (silica gel, hexane/ethyl acetate) affording the pure title compounds 3ac and 3bc. Compound 3ae isomerized during chromatographic purification yielding a ca. 3:1 mixture (300 $\mathrm{MHz}{ }^{1} \mathrm{H}-\mathrm{NMR}$ ) of compounds 3ae:3'ae. Yields are included in Chart 1. Spectroscopic and analytical data follow.

Cyclohexyl 2,2-dimethyl-1-(hydroxypropyl) ketone (3ac). Oil; $R_{\mathrm{f}}=0.55$ (hexane/ethyl acetate:7/3); IR (film) v $3472(\mathrm{OH}), 1700(\mathrm{C}=\mathrm{O}), 1111,1076,1055 \mathrm{~cm}^{-1}$ (CO); ${ }^{1} \mathrm{H}-\mathrm{NMR}$ $\left(\mathrm{CDCl}_{3}\right) \delta 0.98\left[9 \mathrm{H}, \mathrm{s},\left(\mathrm{CH}_{3}\right)_{3} \mathrm{C}\right], 1.10-1.40,1.50-1.90\left(4 \mathrm{H}, 6 \mathrm{H}\right.$, respectively, $\left.2 \mathrm{~m}, 5 \times \mathrm{CH}_{2}\right), 2.60$ $\left(1 \mathrm{H}, \mathrm{tt}, J=11.0,3.05, \mathrm{CHCH}_{2}\right), 3.19(1 \mathrm{H}, \mathrm{d}, J=7.0, \mathrm{OH}), 3.99(1 \mathrm{H}, \mathrm{d}, J=7.0, \mathrm{CHOH}) ;{ }^{13} \mathrm{C}-$ $\operatorname{NMR}\left(\mathrm{CDCl}_{3}\right) \delta 26.3\left[\left(\mathrm{CH}_{3}\right)_{3} \mathrm{C}\right], 25.0,25.7,26.1,26.2530 .35\left(5 \times \mathrm{CH}_{2}\right), 36.05\left[\left(\mathrm{CH}_{3}\right)_{3} \mathrm{C}\right], 49.9$ $\left(\mathrm{CHCH}_{2}\right) 82.1(\mathrm{CHOH}), 216.85(\mathrm{C}=\mathrm{O})$; LRMS m/z $199\left(\mathrm{M}^{+}+1,0.3 \%\right), 198\left(\mathrm{M}^{+}, 0.1 \%\right), 142$ (20), 112 (18), 95 (64), 87 (98), 83 (83), 69 (42), 57 (50), 55 (72), 45 (35); HRMS m/z M+, 198.1616, $\mathrm{C}_{12} \mathrm{H}_{22} \mathrm{O}_{2}$ requires 198.1620 .

Cyclohexyl 1-(hydroxyhexyl) ketone 3ae and cyclohexyl(hydroxymethyl) pentyl ketone ( 3'ae) mixture. Oil; $R_{\mathrm{f}}=0.61$ (hexane/ethyl acetate:7/3); IR (film) $\vee 3485(\mathrm{OH}), 1706(\mathrm{C}=\mathrm{O})$, 1115, 1084, $1063 \mathrm{~cm}^{-1}(\mathrm{CO}) ;{ }^{1} \mathrm{H}-\mathrm{NMR}\left(\mathrm{CDCl}_{3}\right) \delta 0.90\left(6 \mathrm{H}, \mathrm{m}, 2 \times \mathrm{CH}_{3}\right), 1.00-2.00(35 \mathrm{H}, \mathrm{m}, 17$ $\left.\times \mathrm{CH}_{2}, \mathrm{CHCHOH}\right), 2.45\left(2 \mathrm{H}, \mathrm{m}, \mathrm{CH}_{2} \mathrm{CO}\right), 2.54(1 \mathrm{H}, \mathrm{m}, \mathrm{CHCO}), 3.44,3.52(2 \mathrm{H}, 2 \mathrm{br} \mathrm{s}, 2 \times \mathrm{OH})$, $4.04(1 \mathrm{H}$, br s, $\mathrm{CHOHCH}), 4.30(1 \mathrm{H}$, br s, $\mathrm{CHOHCH}) ;{ }^{13} \mathrm{C}-\mathrm{NMR}\left(\mathrm{CDCl}_{3}\right) \delta 13.8,13.95(2 \times$ $\left.\mathrm{CH}_{3}\right), 22.35,22.45,23.25,24.75,25.1$ (2C), 26.6, 25.8 (2C), 25.95, 26.5, 27.4, 29.8, 30.15, 31.35, 31.6, 33.65, $38.15\left(18 \times \mathrm{CH}_{2}\right), 41.3(\mathrm{CHCHOH}), 45.9(\mathrm{CHCO}), 74.95\left(\mathrm{CHOHCH}_{2}\right)$, $80.65(\mathrm{CHOHCH}), 212.35\left(\mathrm{COCH}_{2}\right), 215.35(\mathrm{COCH}) ; \mathrm{LRMS} \mathrm{m} / \mathrm{z} 212\left(\mathrm{M}^{+}, 0.35 \%\right), 142(3), 130$ (1), 111 (12), 95 (11), 83 (100), 55 (84), 43 (17), 41 (52)(first isomer); $\mathrm{m} / \mathrm{z} 194\left(\mathrm{M}^{+}-\mathrm{H}_{2} \mathrm{O}, 0.2 \%\right.$ ), 130 (3), 113 (18), 95 (100), 83 (23), 67 (13), 55 (35), 43 (43) (second isomer).

4-Hydroxy-2,5,5-trimethyl-3-hexanone (3bc). Oil; $R_{\mathrm{f}}=0.57$ (hexane/ethyl acetate:7/3);IR $\left(\right.$ film) $\vee 3484(\mathrm{OH}), 1705(\mathrm{C}=\mathrm{O}), 1120,1095,1077 \mathrm{~cm}^{-1}(\mathrm{CO}) ;{ }^{1} \mathrm{H}-\mathrm{NMR}\left(\mathrm{CDCl}_{3}\right) \delta 0.98[9 \mathrm{H}, \mathrm{s}$, $\left.\left(\mathrm{CH}_{3}\right)_{3} \mathrm{C}\right], 1.09,1.12\left[3 \mathrm{H}, 3 \mathrm{H}\right.$, respectively, $\left.2 \mathrm{~d}, J=4.88,4.88,\left(\mathrm{CH}_{3}\right)_{2} \mathrm{CH}\right], 2.88$ [1H, septet, $J=$ 6.7, $\left.\left(\mathrm{CH}_{3}\right)_{2} \mathrm{CH}\right], 3.15(1 \mathrm{H}, \mathrm{d}, J=7.9, \mathrm{OH}), 4.02(1 \mathrm{H}, \mathrm{d}, J=7.9, \mathrm{CHOH}) ;{ }^{13} \mathrm{C}-\mathrm{NMR}\left(\mathrm{CDCl}_{3}\right) \delta$ 16.4, $19.9\left[\left(\mathrm{CH}_{3}\right)_{2} \mathrm{C}\right], 25.75\left[\left(\mathrm{CH}_{3}\right)_{3} \mathrm{C}\right], 35.95\left[\left(\mathrm{CH}_{3}\right)_{3} \mathrm{C}\right], 39.55\left[\left(\mathrm{CH}_{3}\right)_{2} \mathrm{CH}\right], 82.05(\mathrm{CHOH})$, $217.85(\mathrm{C}=\mathrm{O})$; LRMS m/z $158\left(\mathrm{M}^{+}, 0.2 \%\right), 102$ (40), 87 (100), 73 (17), 72 (22), 71 (24), 69 (54), 57 (56), 55 (15), 45 (53), 43 (83).

Methyl 2-cyclohexyl-2-oxoacetate (3ag). Once $\alpha$-chloroenamine 1a was lithiated as it was above described (Method A), a stream of carbon dioxide was passed for $10 \mathrm{~min}$ at $-78{ }^{\circ} \mathrm{C}$ 
allowing the temperature to rise to $-40^{\circ} \mathrm{C}$. Then, the resulting mixture was hydrolyzed with water, acidified with $2 \mathrm{M}$ hydrochloric acid and worked up as described already for compound 2aa. The residue obtained was dissolved in methanol with two drops of concentrated sulfuric acid and the mixture was refluxed for $1 \mathrm{~h}$. The resulting mixture was treated with a saturated solution of sodium chloride and extracted with ethyl acetate. The organic layer was washed with water, dried over anhydrous $\mathrm{Na}_{2} \mathrm{SO}_{4}$ and evaporated (15 Torr). The residue was purified by column chromatography (silica gel, hexane/ethyl acetate) yielding the title compound 3ag as an oil. Yield is given in Chart 1. $R_{\mathrm{f}}=0.33$ (hexane/ethyl acetate:9/1); IR (film) $v 1728 \mathrm{~cm}^{-1}(\mathrm{C}=\mathrm{O}$ ); ${ }^{1} \mathrm{H}-\mathrm{NMR}\left(\mathrm{CDCl}_{3}\right) \delta 1.15-1.40,1.65-1.95\left(6 \mathrm{H}, 4 \mathrm{H}\right.$, respectively, $\left.2 \mathrm{~m}, 5 \times \mathrm{CH}_{2}\right), 3.04(1 \mathrm{H}, \mathrm{m}, \mathrm{CH})$, $3.86\left(3 \mathrm{H}, \mathrm{s}, \mathrm{CH}_{3}\right) ;{ }^{13} \mathrm{C}-\mathrm{NMR}\left(\mathrm{CDCl}_{3}\right) \delta 25.2(2 \mathrm{C}), 25.6,27.4(2 \mathrm{C})\left(5 \times \mathrm{CH}_{2}\right), 46.25(\mathrm{CH}), 52.65$ $\left(\mathrm{CH}_{3}\right), 162.2\left(\mathrm{CO}_{2}\right), 197.15(\mathrm{C}=\mathrm{O})$; LRMS m/z $142\left(\mathrm{M}^{+}, 18 \%\right), 113$ (16), 111 (12), 110 (16), 87 (76), 83 (52), 82 (21), 74 (38), 67 (21), 55(100).

DTBB-Catalyzed lithiation of $\alpha$-chloroenamines 1 and reaction with chalcone or cyclohexyl isocyanate (Method A). Isolation of compounds 3'af, 3ah and $3 \mathrm{bh}$. General procedure

Once lithiation of starting $\alpha$-chloroenamines was carried out as it was above described (Method A), the corresponding organolithium intermediate was treated with a $1: 1$ precooled $\left(-40{ }^{\circ} \mathrm{C}\right)$ mixture of chalcone and $\mathrm{BF}_{3} \cdot \mathrm{OEt}_{2}$ or cyclohexyl isocyanate allowing the temperature to rise to $40{ }^{\circ} \mathrm{C}$. After hydrolyzing with water, the resulting mixture was acidified with $2 \mathrm{M}$ hydrochloric acid, stirred for $1 \mathrm{~h}$ and neutralized with $3 \mathrm{M}$ sodium hydroxide before the final work up as it was above described for compound 2aa. Thus, title compounds $\mathbf{3}$ were isolated, their yields being included in Chart 1. Spectroscopic and analytical data follow.

Cyclohexyl 1,3-diphenyl-3-oxopropyl ketone (3'af). Oil; $R_{\mathrm{f}}=0.18$ (hexane/ethyl acetate:8/2); IR (film) v 1723, $1686 \mathrm{~cm}^{-1}(\mathrm{C}=\mathrm{O}) ;{ }^{1} \mathrm{H}-\mathrm{NMR}\left(\mathrm{CDCl}_{3}\right) \delta 1.00-1.75\left(10 \mathrm{H}, \mathrm{m}, 5 \times \mathrm{CH}_{2}\right.$ cyclohexyl ring), 2.40-2.50 (1H, m, CHCO), $3.00(1 \mathrm{H}, \mathrm{dd}, J=18.0,3.7, \mathrm{CHHCO}), 3.95(1 \mathrm{H}$, dd, $J=18.0,10.4, \mathrm{CHHCO}), 4.50(1 \mathrm{H}, \mathrm{dd}, J=10.4,3.7, \mathrm{CHPh}), 7.10-7.50$ (8H, m, ArH), 7.89 (2H, $\mathrm{d}, J=7.4, \mathrm{ArH}) ;{ }^{13} \mathrm{C}-\mathrm{NMR}\left(\mathrm{CDCl}_{3}\right) \delta 25.4,25.8,25.9,28.4,29.45\left(5 \times \mathrm{CH}_{2}\right.$ cyclohexyl ring $)$, $42.75\left(\mathrm{CH}_{2} \mathrm{CO}\right), 50.0\left[\mathrm{CH}\left(\mathrm{CH}_{2}\right)_{2}\right], 51.8(\mathrm{CHPh}), 127.4,128.1,128.4,128.5,129.05,133.15$, 136.6, $138.25\left(8 \times\right.$ ArC), $198.2(\mathrm{PhCO}), 212.25(\mathrm{CO}) ; \mathrm{LRMS}$ m/z $320\left(\mathrm{M}^{+}, 0.5 \%\right), 111(21), 105$ (47), 83 (100), 77 (31), 55 (47), 41 (28).

$N$,2-Dicyclohexyl-2-oxoacetamide (3ah). Oil; $R_{\mathrm{f}}=0.56$ (hexane/ethyl acetate:7/3); IR (film) v $3317(\mathrm{NH}), 1711(\mathrm{C}=\mathrm{O}), 1653,1530 \mathrm{~cm}^{-1}(\mathrm{NC}=\mathrm{O}) ;{ }^{1} \mathrm{H}-\mathrm{NMR}\left(\mathrm{CDCl}_{3}\right) \delta 1.10-1.50,1.60-2.00$ $\left(10 \mathrm{H}, 10 \mathrm{H}, 2 \mathrm{~m}, 10 \times \mathrm{CH}_{2}\right), 3.40(1 \mathrm{H}, \mathrm{tt}, J=11.0,3.0, \mathrm{CHCO}), 3.65-3.80(1 \mathrm{H}, \mathrm{m}, \mathrm{CHNH}), 6.81$ $\left(1 \mathrm{H}\right.$, br s, NH); ${ }^{13} \mathrm{C}-\mathrm{NMR}\left(\mathrm{CDCl}_{3}\right) \delta 24.65(2 \mathrm{C}), 25.35(3 \mathrm{C}), 25.75,28.1(2 \mathrm{C}), 32.6(2 \mathrm{C})(10 \times$ $\left.\mathrm{CH}_{2}\right), 43.25(\mathrm{CHCO}), 48.25(\mathrm{CHN}), 158.9(\mathrm{NC}=\mathrm{O}), 201.95(\mathrm{C}=\mathrm{O})$; LRMS m/z $237\left(\mathrm{M}^{+}, 15 \%\right)$, 111 (20), 83 (100), 55 (60), 43 (11), 41 (49).

$N$-Cyclohexyl-3-methyl-2-oxobutanamide (3bh). Oil; $R_{\mathrm{f}}=0.52$ (hexane/ethyl acetate:7/3); IR (film) v $3274(\mathrm{NH}), 1720(\mathrm{C}=\mathrm{O}), 1673,1650,1530 \mathrm{~cm}^{-1}(\mathrm{NC}=\mathrm{O}) ;{ }^{1} \mathrm{H}-\mathrm{NMR}\left(\mathrm{CDCl}_{3}\right) \delta 1.13(6 \mathrm{H}$, $\left.\mathrm{d}, J=6.7,2 \times \mathrm{CH}_{3}\right), 1.10-1.50,1.60-2.00\left(5 \mathrm{H}, 5 \mathrm{H}, 2 \mathrm{~m}, 5 \times \mathrm{CH}_{2}\right), 3.62[1 \mathrm{H}$, sept, $J=6.7$, $\left.\mathrm{CH}\left(\mathrm{CH}_{3}\right)_{2}\right], 3.70-3.80(1 \mathrm{H}, \mathrm{m}, \mathrm{CHNH}), 6.87\left(1 \mathrm{H}\right.$, br s, NH); ${ }^{13} \mathrm{C}-\mathrm{NMR}\left(\mathrm{CDCl}_{3}\right) \delta 17.75(2 \mathrm{C}, 2 \times$ $\left.\mathrm{CH}_{3}\right), 24.65(2 \mathrm{C}), 25.35,32.65(2 \mathrm{C})\left(5 \times \mathrm{CH}_{2}\right), 33.9(\mathrm{CHC}), 48.25(\mathrm{CHN}), 158.8(\mathrm{NC}=\mathrm{O}), 202.8$ 
$(\mathrm{C}=\mathrm{O})$; LRMS m/z $197\left(\mathrm{M}^{+}, 20 \%\right), 126$ (18), 83 (100), 71 (25), 55 (82), 43 (73); HRMS M+, 197.1412, $\mathrm{C}_{11} \mathrm{H}_{19} \mathrm{NO}_{2}$ requires 197.1416.

\section{Acknowledgements}

Finacial support by the DGES of the Spanish Ministerio de Educación y Cultura (MEC no. PB97-0123 and PB97-0133) is gratefully acknowledged.

\section{References and Notes}

1. For a review, see: Nájera, C.; Yus, M. Org. Prep. Proc. Int. 1995, 27, 383.

2. Seebach, D. Angew. Chem., Int. Ed. 1979, 18, 239.

3. For leading references, see: (a) Trzupck, L. S.; Newirth, T. L.; Kelly, E. G.; Sbarbati, N. E.; Whitesides, G. M. J. Am. Chem. Soc. 1973, 95, 8118. (b) Nudelman, N. S.; Doctorovich, F.; Amorin, G. Tetrahedron Lett. 1990, 31, 2553. (c) Nudelman, N. S.; Doctorovich, F. Tetrahedron 1994, 50, 4651. (d) Nudelman, N. S.; Doctorovich, F. J. Chem. Soc., Perkin Trans. 2 1994, 1233. (e) Nudelman, N. S.; Vitale, A. A. Org. Prep. Proc. Int. 1981, 13, 144. (f) Nudelman, N. S.; Outumuro, P. J. Org. Chem. 1982, 47, 4347. (g) Seyferth, D.; Weinstein, R. M. J. Am. Chem. Soc. 1982, 104, 5534. (h) Seyferth, D.; Weinstein, R. M.; Wang, W. L. J. Org. Chem. 1983, 48, 1144. (i) Hui, R. C.; Seyferth, D. Org. Synth. Coll. 1990, Vol. VIII, 346, and references cited therein.

4. Hiiro, T.; Morita, Y.; Inone, T.; Kambe, N.; Ogawa, A.; Ryu, I.; Sonoda, N. J. Am. Chem. Soc. 1990, 112, 455.

5. Shiner, C. S.; Berks, A. H.; Fisher, A. M. J. Am. Chem. Soc. 1988, 110, 957.

6. For reviews see, for instance: (a) Gröbel, B. T.; Seebach, D. Synthesis 1977, 357. (b) Ager, D. J. In Umpoled Synthons, Hase, T. A., Ed; J. Wiley \& Sons: New York, 1987; p. 19. (c) Krief, A. In Comprehensive Organic Synthesis, Trost, B. M.; Fleming, I,; Pattenden, G., Eds.; Pergamon: New York, 1993; Vol. 3, p. 121. (d) Vallée, Y.; Bulpin, A. In Comprehensive Organic Functional Group Transformations, Katritzky, A. R.; Meth-Cohn, O.; Rees, C. W.; Kirby, G. W., Eds.; Pergamon: New York, 1995; Vol. 4, p 243.

7. For the preparation of imidoyl lithium intermediates by chlorine-lithium exchange, see: (a) Alonso, E.; Ramón, D. J.; Yus, M. Tetrahedron Lett. 1997, 38, 8903. (b) Alonso, E.; Ramón, D. J.; Yus, M. Tetrahedron 1998, 54, 12007.

8. For the preparation of carbamoyl and thiocarbamoyl lithium reagents by chlorine-lithium exchange, see: (a) Ramón, D. J.; Yus, M. Tetrahedron Lett. 1993, 34, 7115. (b) Ramón, D. J.; Yus, M. Tetrahedron 1996, 52, 13739.

9. For reviews, see: (a) See references 1 and 6b. (b) See, also: Kolb, M. In Umpoled Synthons, Hase, T. A., Ed.; J. Wiley \& Sons: New York, 1987; p 73. 
10. For leading references see, for instance: (a) Baldwin, J. E.; Höfle, G. A.; Lever, O. W. J. Am. Chem. Soc. 1974, 96, 7125. (b) Levy, A. B.; Schwartz, S. J. Tetrahedron Lett. 1976, 2210. (c) Chavdarian, C. G.; Heathcock, C. H. J. Am. Chem. Soc. 1975, 97, 3822. (d) Baldwin, J. E.; Lever, O. W.; Tzodikov, N. R. J. Org. Chem. 1976, 41, 2312. (e) Schöllkopf, U.; Hänssle, P. Justus Liebigs Ann. Chem. 1972, 763, 208. (f) Dexheimer, E. M.; Spielter, L. J. Organomet. Chem. 1976, 107, 229. (g) Boeckman, R. K.; Bruza, K. J.; Baldwin, J. E.; Lever, O. W. J. Chem. Soc., Chem. Commun. 1975, 519. (h) Eis, M. J.; Wrobel, J. E.; Ganem, B. J. Am. Chem. Soc. 1984, 106, 3693.

11. First account: Yus, M.; Ramón, D. J. J. Chem. Soc., Chem. Commun. 1991, 398.

12. Mechanistic studies: Yus, M.; Herrera, R. P.; Guijarro, A. Tetrahedron Lett. 2001, 42, 3455.

13. For a polymer-supported arene-catalyzed version of this reaction, see: (a) Gómez, C.; Ruiz, S.; Yus, M. Tetrahedron Lett. 1998, 39, 1397. (b) Gómez, C.; Ruiz, S.; Yus, M. Tetrahedron 1999, 55, 7017.

14. For reviews, see: (a) Yus, M. Chem. Soc. Rev. 1996, 155. (b) Ramón, D. J.; Yus, M. Eur. J. Org. Chem. 2000, 225 (Microreview). (c) Yus, M. Synlett 2001, 1197 (Account).

15. (a) For a review, see: Guijarro, D.; Yus, M. Recent Res. Devel. Org. Chem. 1998, 2, 713. (b) Last paper on this topic from our laboratory: Guijarro, D.; Yus, M. J. Organomet. Chem. 2001, 624, 53.

16. For reviews, see: (a) Nájera, C.; Yus, M. Trends Org. Chem. 1991, 2, 155. (b) Nájera, C.; Yus, M. Recent. Res. Devel. Org. Chem. 1997, 1, 67.

17. Last paper on this topic from our laboratory: Yus, M.; Soler, T.; Foubelo, F. J. Org. Chem. 2001, 66, 6207.

18. (a) For a review, see: Yus, M.; Foubelo, F. Rev. Heteroatom Chem. 1997, 17, 73. (b) Last paper on this topic from our laboratory: Yus, M.; Foubelo, F.; Ferrández, J. V. Eur J. Org. Chem. 2001, 2809.

19. (a) For a review, see: Foubelo, F.; Yus, M. Trends Org. Chem. 1998, 7, 1. (b) Last paper on this topic from our laboratory: Yus, M.; Gutierrez, A.; Foubelo, F. Tetrahedron 2001, 57, 4411.

20. For a review, see: Guijarro, A.; Gómez, C.; Yus, M. Trends Org. Chem. 2000, 8, 65.

21. Last paper on this topic from our laboratory: Yus, M.; Radivoy, G.; Alonso, F. Synthesis 2001, 914.

22. Review: Alonso, F.; Yus, M. Recent Res. Devel. Org. Chem. 1997, 1, 397.

23. For a review on $\alpha$-heteroatom-substituted alkenyllithium reagents, including a few examples of stabilized $\alpha$-lithioenamines, see: Braun, M. Angew. Chem., Int. Ed. 1998, 37, 430.

24. Ghosez, L.; George-Koch, I.; Patiny, L.; Houteky, P. B.; Nshimyumukiza, P.; Phan, T. Tetrahedron 1998, 54, 9207.

25. For $\mathrm{BF}_{3}$ activation of $\alpha, \beta$-unsaturated carbonyl compounds in the reaction with functionalized organolithium compounds, see: Yus, M.; Pastor, I. M.; Gómis, J. Tetrahedron 2001, 57, 5799.

26. Ramón, D. J.; Yus, M. Tetrahedron: Asymmetry 1997, 8, 2479. 